\title{
WhatsOnWeb: Using Graph Drawing to Search the $\mathrm{Web}^{\star}$
}

\author{
Emilio Di Giacomo, Walter Didimo, Luca Grilli, and Giuseppe Liotta \\ Università di Perugia, Italy \\ \{digiacomo, didimo, grilli, liotta\}@diei.unipg.it
}

\begin{abstract}
One of the most challenging issues in mining information from the World Wide Web is the design of systems that can present the data to the end user by clustering them into meaningful semantic categories. We envision that the analysis of the results of a Web search can significantly take advantage of advanced graph drawing techniques. In this paper we strengthen our point by describing the visual functionalities of WhatsOnWeb, a meta search clustering engine explicitly designed to make it possible for the user to browse the Web by means of drawings of graphs whose nodes represent clusters of coherent data and whose edges describe semantic relationships between pairs of clusters. A prototype of WhatsOnWeb is available at http://whatsonweb.diei.unipg.it/.
\end{abstract}

\section{Introduction}

Increasing attention has been recently devoted to the development of new systems that support users in searching the Web. Although classical search engines perform well in many circumstances, there are some practical situations in which the data that they return as a reply to a user's query are not structured enough to easily convey the information that the user may be looking for (see also [6, 10]). Indeed, the output of a classical search engine consists of an ordered list of document links (URLs), where each link comes together with a brief summary (called snippet) of its associated document. The links are selected and ranked by the search engine according to some criteria that depend on the user's query, on the documents content, and (in some cases, like Google) on the popularity of the links in the World Wide Web. The list of URLs returned by a search engine is often very long and the consequence is that users may omit to check URLs that can be relevant for them, just because these links do not appear in the first positions of the list. This problem is even more evident when the user's query presents polisemy, that is words with different meanings. For example, suppose that the user submits the query "Jaguar"; is she interested in the "car" or in the "animal"?

A Web meta-search clustering engine is a system conceived to cope with the above described limitations of classical search engines. As shown in Figure 1, a

\footnotetext{
* This work is partially supported by the MIUR Project ALGO-NEXT: Algorithms for the Next Generation Internet and Web: Methodologies, Design and Applications.
} 


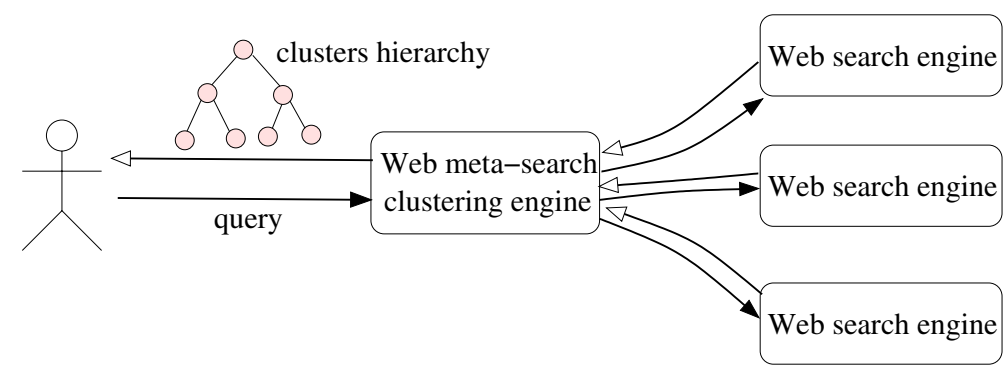

Fig. 1. Schema of the working model of a Web meta-search clustering engine

Web meta-search clustering engine provides a visual interface to the user who submits a query; it forwards the query to (one or more) traditional search engines, and it displays a set of clusters, also called categories, which are typically organized in a hierarchy. Each category contains URLs of documents that are semantically related with each other and is labelled with a string that describes its content. With this approach, the user has a global view of the different semantic areas involved by her query, and can explore those areas in which she is mostly interested.

Despite the graphical user interface of a Web meta-search clustering engines plays a fundamental role for efficiently retrieving the wanted information, the vast majority of Web meta-search clustering engines (see, e.g., Vivisimo, iBoogie, SnakeT [6] 1] ) have a GUI in which the set of clusters is represented simply as a tree of directories and subdirectories. We find this type of representation unsatisfactory for the following reasons.

- A tree of clusters represents inclusion relationships between a cluster and its sub-clusters. However, nodes that are not in an ancestor/descendant relation can also have strong semantic connections, which may be underestimated if not completely lost by following the tree structure. Suppose for example that the user's query is "Armstrong" and that the tree of clusters is as the one depicted in Figure 2(a). Is the category "Biography" related to "Louis" or to "Lance" or to both (or to neither of them but to the astronaut Neil Armstrong?). If there were one edge as in Figure 2(b) or if there were two edges with different weights as in Figure 2(c) the user could decide whether or not the category "Biography" is of her interest.

- Viewing the clusters as the vertices of a graph can also help the user to detect communities of clusters which identify a common topic and/or to highlight isolated clusters which may be pruned if their labels are not meaningful for the user's query. This type of analysis is clearly unfeasible if all clusters are displayed in a tree structure.

- Finally, the visualization paradigm adopted by systems like Vivisimo often uses basic drawing techniques: The tree is displayed with poor aspect ratio on the left-hand side of the interaction window. The visual exploration is

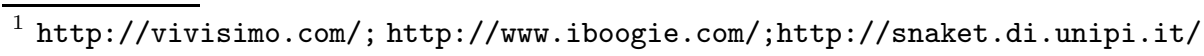




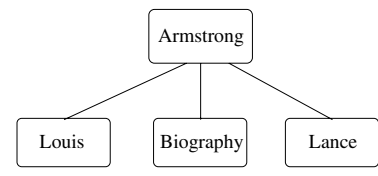

(a)

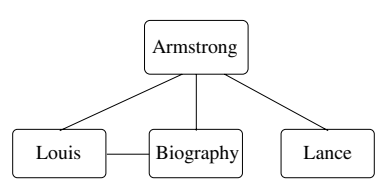

(b)

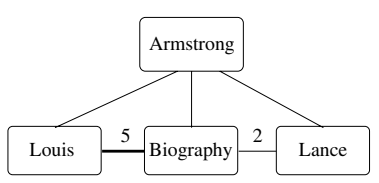

(c)

Fig. 2. (a) A portion of a tree of categories for the query "Armstrong". (b)-(c) The same tree, plus edges that highlight cluster relationships. In (c), each edge is labelled with a number that represents the strength of the edge.

particularly uncomfortable when the degree of the tree is high: Expanding a few nodes at the same level can make the rest of the tree no longer visible on the screen.

We envision that the analysis of the results of a Web meta search clustering engine can significantly take advantage of advanced graph drawing techniques. In this paper we strengthen our point by describing the user interface of WhatsOnWeb2. WhatsOnWeb, a meta search clustering engine explicitly designed to make it possible for the user to browse the Web by means of drawings of graphs whose nodes represent clusters of coherent data and whose edges describe semantic relationships between pairs of clusters. The produced diagrams are in general structurally more complex than trees and the user can interact with them in a number of different ways. The main visual functionalities and tools of the GUI of WhatsOnWeb are shortly summarized below.

- Whats0nWeb adopts a visual paradigm that makes it possible to dynamically interact with the graph of categories by expanding and/or contracting its nodes while preserving the user's mental-map. Particular attention is given to the area required by the representation, since the graph of the clusters may become relatively large after the expansion of a few categories.

- Whats0nWeb emphasizes the relevance of a category with respect to other categories by using a suitable score function. The relevance (score) of each category is conveyed in the drawing by using different colors for the vertices of the graph. It is also possible to visualize the "strength" of a semantic relationship between two categories expressed as another suitable score function. Furthermore, the user can analyze the relationship between two categories $c_{1}$ and $c_{2}$ by visualizing a bipartite graph with all URLs that give rise to the relationship between $c_{1}$ and $c_{2}$.

- The user is provided with several automatic or semiautomatic tools to simplify and/or to refine the clustered graph returned by the system. For example, the user can ask the system to prune those categories whose relevance is below a given threshold and that are not connected to any other relevant categories.

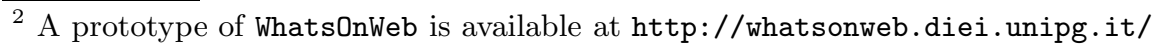


We remark that, to the best of our knowledge, the only other Web meta-search clustering engine that supports the user with a graph visualization approach is Kartoo 3. Besides being very appealing from a graphic view point, the graph visualization offered by Kartoo is however not much more expressive than the tree of clusters of Vivisimo. The nodes in the graph of Kartoo are the individual URLs and the clusters are represented as regions of a virtual map. By pointing the page with the mouse, the system highlights all categories in which the Web page is contained; by pointing a category, the system highlights all URLs that form the specified category. Also, every map of Kartoo contains only a limited number of URLs (about twelve) and a global view of the relationships between all categories (and thus an enhanced visual analysis) is not possible.

\section{Principles of the System WhatsOnWeb}

The system WhatsOnWeb computes the clusters hierarchy based on the topologydriven approach [5], which allows us to define a new visualization paradigm for representing and browsing the clusters hierarchy. The topology-driven approach relies on the basic concept of snippet graph. Intuitively, a snippet graph represents the URLs returned by a search engine in response to a user query, along with their relationships. The relationships between pairs of URLs are computed by analyzing the snippet that a search engine returns along with each URL; the snippet of a URL is a text that represents a brief summary of the document associated with the URL. Each vertex of the snippet graph is a URL and there is an edge between two URLs when the associated snippets share some terms. In the following, we first give a formal definition of snippet graph, which is refined with respect to that introduced in [5]; then, we recall the principles of a visualization paradigm related to the definition of a snippet graph.

Let $U$ be a set of URLs returned by a search-engine in response to a user query. If $u \in U$, we call text of $u$ the concatenation of the title and the snippet associated with $u$. In order to construct the snippet graph of $U$ we perform a sequence of preliminary steps:

Cleaning step. Stop-words and HTML symbols are removed in the text of each element of $U$.

Stemming step. A stemming algorithm is applied on the cleaned texts, and for every computed stem $s$ we associate a word $z_{s}$ chosen among those whose stem is equal to $s$; words $z_{s}$ is used later to label the clusters. After this step, the text of each URL $u$ is viewed as a sequence of stems, denoted by $S_{u}$.

Scoring step. We assign a score $w_{s}$ to each stem $s ; w_{s}$ measures the "relevance" of $s$ in all URL texts containing $s$. Several criteria can be used to determine $w_{s}$. We adopt a standard function called $t f$-idf (term frequency-inverse document frequency), introduced by Salton in 1989 [9. Namely, let $D$ be a set of documents, let $s$ be a term in a document $d \in D$, and let $D_{s} \subseteq D$ be the subset of documents containing $s$. The tf-idf of $s$ with respect to document

$\overline{3}$ http://www.kartoo.com/ 
$d$ is defined as: $t(s, d)=f(s, d) \log \left(|D| /\left|D_{s}\right|\right)$, where $f(s, d)$ is the frequency of $s$ in $d$. In our case, assuming $D$ as the set of URL texts, we define $w_{s}$ as the average of the tf-idf of $s$ over all documents of $D_{s}$, i.e., $w_{s}=\frac{\sum_{d \in D_{s}} t(s, d)}{\left|D_{s}\right|}$.

The snippet graph $G$ of $U$ is a labeled weighted graph defined as follows:

- $G$ has a vertex $v_{u}$ associated with each element $u \in U$. The label of $v_{u}$ can be either the title of $u$ or its description as URL.

- $G$ has an edge $e=\left(v_{u_{1}}, v_{u_{2}}\right)$ (where $u_{1}, u_{2} \in U$ ) if the texts of $u_{1}$ and $u_{2}$ share some stems, i.e. if $S_{u_{1}} \cap S_{u_{2}}$ is not empty. To determine the weight and the label of $e$, the following procedure is applied. Compute the set $\mathcal{S}_{e}$ of all maximal sub-sequences of consecutive stems (each sub-sequence with single multiplicity) shared by $S_{u_{1}}$ and $S_{u_{2}}$; each element of $\mathcal{S}_{e}$ is also called a sentence. The score of a sentence $\sigma \in \mathcal{S}_{e}$ is defined as $w_{\sigma}=\sum_{s \in \sigma} w_{s}$, and the weight of $e$ is $w_{e}=\sum_{\sigma \in \mathcal{S}_{e}} w_{\sigma}$. Finally, the label of $e$ is set equal to $\mathcal{S}_{e}$.

In the snippet graph, the weight of an edge measures the strength of the semantic relationship between their end-vertices. This strength depends both on the number of sub-sequences of stems shared by their texts and on the relevance (scores) of these sub-sequences. As also pointed out by other authors (see e.g. [6, 11]), considering sub-sequences of terms (i.e., sentences) shared by two texts is in general more informative than considering unordered sets of terms, and it allows better estimation of the strength of the connections between the two texts. Also, sentences can be used to compute more effective labels for clusters.

In order to determine clusters in the snippet graph we compute communities of vertices, i.e. sets of vertices that are strongly connected from a topological point of view. We adopt a recursive divisive strategy based on graph connectivity (see, e.g. [2, 7, 8]): The clusters hierarchy is determined by recursively cutting some edges that disconnect the graph; the algorithms based on this strategy mainly differ for the criteria used to choose the next edge to be removed. Our algorithm is a variation of an elegant technique recently proposed by Brinkmeier [2] (see 5 for details). We remark here that for any pair of categories $A$ and $B$ in the computed hierarchy, we have that either $A$ and $B$ are disjoint or one of them includes the other. In fact, in order to simplify the information returned to the user, we aim at assigning each URL to its most representative category, and possibly relationships between disjoint categories will be highlighted using the new visualization paradigm.

To label a category $\mu$ of our hierarchy tree, we apply a procedure that takes advantage of the new definition of the snippet graph. Namely, let $G_{\mu}=\left(V_{\mu}, E_{\mu}\right)$ be the subgraph of $G$ induced by the vertices of $G$ contained in $\mu$, and let $\mathcal{S}_{\mu}$ be the set of all labels of the edges of $G_{\mu}$, that is $\mathcal{S}_{\mu}=\cup_{e \in E_{\mu}} \mathcal{S}_{e}$. If $\sigma \in \mathcal{S}_{\mu}$ occurs in the label of $k$ edges $(k>0)$ of $G_{\mu}$, then $k w_{\sigma}$ is called the total score of $\sigma$ in $G_{\mu}$. Denoted by $\sigma=s_{1}, s_{2}, \ldots, s_{h}(h>0)$ any sentence of $S_{\mu}$ with maximum total score, the label of $\mu$ is defined as the concatenation of the (spaced) words $z_{s_{1}}, z_{s_{2}}, \ldots, z_{s_{h}}$. 
Let $G$ be the snippet graph of a set of URLs, and $H(G)$ its category tree. For any internal node $\mu$ of $H(G)$, we denote by $G_{\mu}$ the subgraph of $G$ induced by the leaves of the subtree rooted at $\mu$. The drawing approach works in three steps.

Edge sparsification. A preprocessing step is applied on $G$ to remove those edges that do not provide useful information to the structure of the clusters. For each internal node $\mu$ of $H(G)$, we remove from $G_{\mu}$ every edge whose label only consists of sentences that, in their unstemmed version, are completely contained in the label of $\mu$. Denote by $G^{\prime}$ the graph obtained from $G$ by applying this cleaning operation over all sub-graphs $G_{\mu}$. We maintain on $G^{\prime}$ the same clusters hierarchy as $G$, so that $H\left(G^{\prime}\right)=H(G)$ in the following.

Cluster relationships computation. We transform graph $G^{\prime}$ into a new super clustered graph $G^{\prime \prime}$ such that each cluster $\mu$ is explicitly represented as a kind of super node, which we denote by $C_{\mu}$. Super nodes can be connected by super edges to emphasize the relationships among clusters. Super edges can be seen as extra arcs connecting nodes of $H\left(G^{\prime}\right)$. More formally (see also Figures $3(\mathrm{a})$ and $3(\mathrm{~b})$, let $\mu_{1}$ and $\mu_{2}$ be any two internal nodes of $H\left(G^{\prime}\right)$ that are children of the same node $\mu$, and let $E\left(\mu_{1}, \mu_{2}\right)$ be the subset of edges of $G^{\prime}$ that connect vertices of $G_{\mu_{1}}^{\prime}$ to vertices of $G_{\mu_{2}}^{\prime}$. If $E\left(\mu_{1}, \mu_{2}\right)$ is not empty, we replace this set of edges by a new super edge $e$ connecting the super nodes $C_{\mu_{1}}$ and $C_{\mu_{2}}$. The label of $e$ is the union of all labels of the

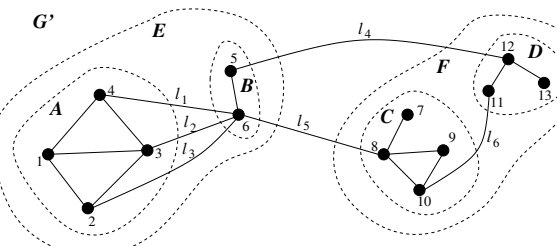

(a)

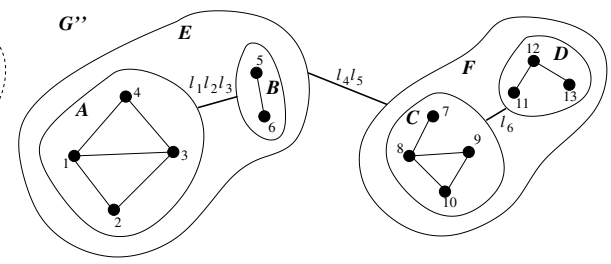

(b)

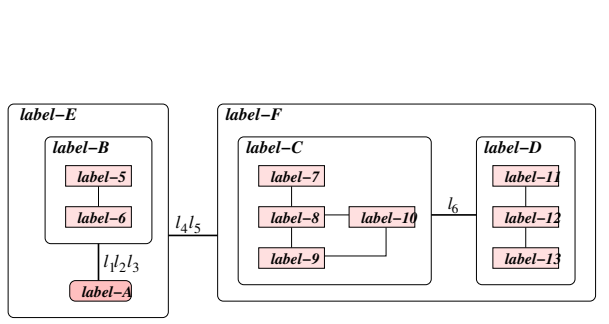

(c)

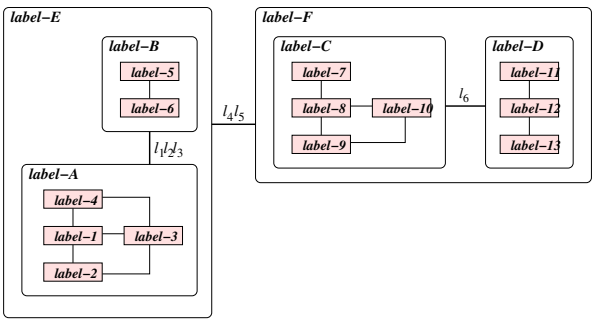

(d)

Fig. 3. (a) A clustered graph $G^{\prime}$ with no redundant edges; the edges connecting vertices in distinct clusters are labelled. (b) The graph $G^{\prime \prime}$ obtained from $G^{\prime}$ by representing clusters with super nodes. (c) A drawing of $G^{\prime \prime}$; the string label-x denotes the label of object (cluster or vertex) $x$; cluster $A$ is contracted.(d) A drawing of $G^{\prime \prime}$ where all clusters are expanded. 
edges in $E\left(\mu_{1}, \mu_{2}\right)$, and the weight of $e$ is the sum of all weights of the edges in $E\left(\mu_{1}, \mu_{2}\right)$.

Maps Visualization. Let $\mu$ be a cluster of $G^{\prime \prime}$. The graph consisting of the children of $\mu$ and their relationships is represented as an orthogonal drawing with box-vertices; each vertex is drawn as a box with the size required to host its label or its subclusters, depending on the fact that it is contracted or expanded. Figures 3(c) and 3(d) show two different maps of the clustered graph $G^{\prime \prime}$ in Figure $3(\mathrm{~b})$, Details about the algorithms used to compute the drawings are given in Section 4

\section{User Interface and Graph Visualization Functionalities}

The user interacts with the system Whats0nWeb by means of a Web browser and a Java applet. The graphic environment consists of two frames (see, e.g., Figure 4). In the left frame the clusters hierarchy is presented to the user as a classical directories tree. In the right frame, the user interacts with a map that gives a graphical view of the clusters hierarchy at the desired level of abstraction. Initially, the map shows only the first level categories and their relationships, according to the principles given in the previous section. The drawing is computed using an orthogonal drawing style, where each category is represented as a box with prescribed size. The size of each box is chosen as the minimum required to host the label of the category and/or a drawing of its subclasters. The user can decide to expand any of these categories, by simply clicking on the corresponding box, and so on recursively. Expanded categories can also be contracted by the user successively. Figure 4 shows a snapshot of the interface, where the results for the query "Armstrong" are presented; in the figure, the category "Louis Armstrong" has been expanded by the user. During the browsing, the system automatically keeps consistent the map and the tree, i.e., if a category is expanded in the map, it also appears expanded in the tree, and viceversa. Also, in order to preserve the user mental map, WhatsOnWeb preserves the orthogonal shape of the drawing during any expansion or contraction operation. For example, Figure $5(\mathrm{a})$ shows the map obtained by expanding the categories "Jazz", "School", and "Louis Armstrong Stamp" in the map of Figure 4.

Besides the browsing functionalities above described, the interface is equipped with several facilities that increase the expressiveness of the output and the interaction between the system and the user. They are listed and described below:

- Ranking of categories. WhatsOnWeb ranks the semantic categories at each level, by assigning to each category a relevance score. This score is proportional to these three main parameters: (a) the size of the category, (b) the maximum rank (returned by Google) of a document in the category, and (c) the deviation of the score for the label of the category from the average score of all sentences in the same category. We use this third parameter as an estimate of the reliability of the sentence used to label the category. In the tree 


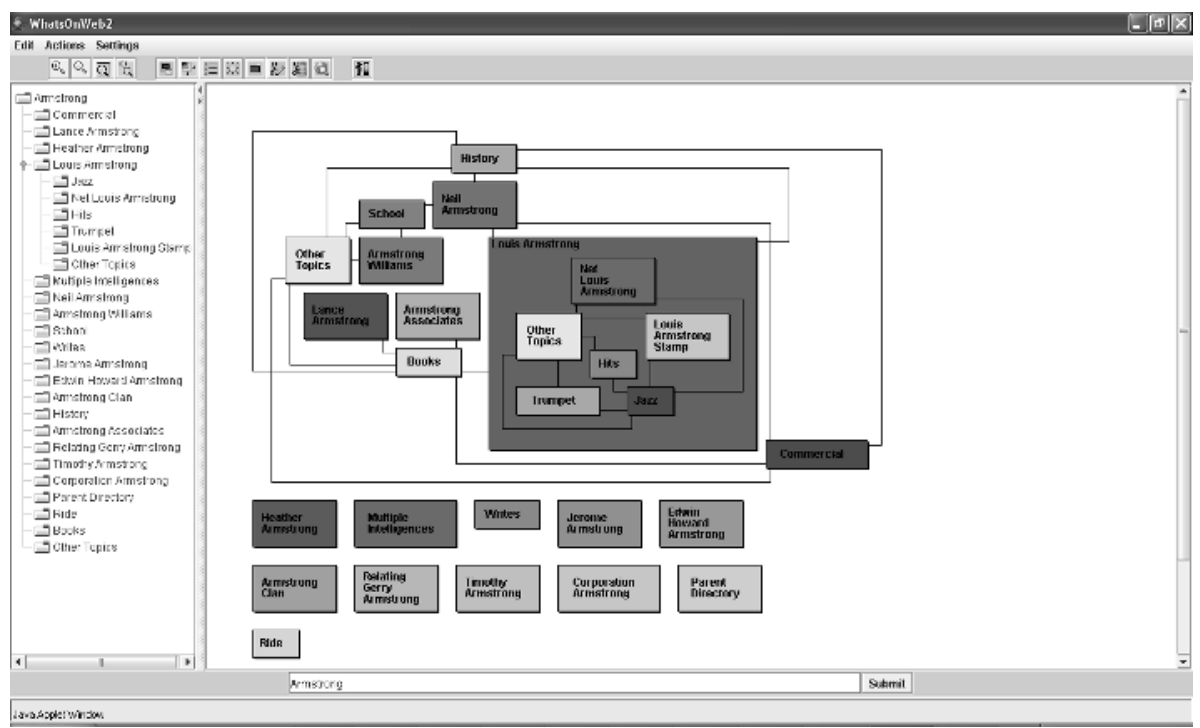

Fig. 4. A map for the query "Armstrong"; in the map the category "Louis Armstrong" has been expanded by the user

representation, the categories are ordered from top to bottom according to their decreasing relevance score. In the map, the relevance score is conveyed to the user adopting different colors in the red scale; highly red categories are the most relevant ones, while yellow categories are the least relevant. For example, in Figure 4, the expanded category "Louis Armstrong" is supposed to be one of the most relevant categories at the first level. Conversely, the category "Ride" has a low relevance score for the system, and its label does

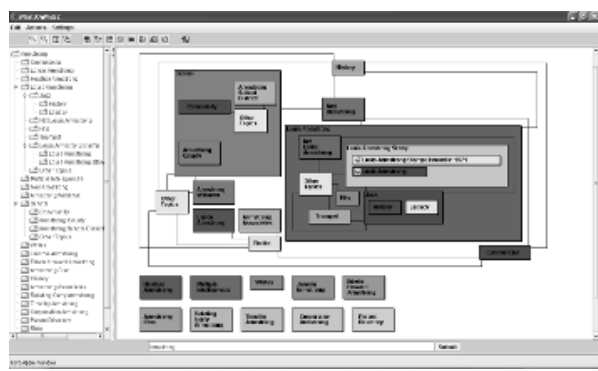

(a)

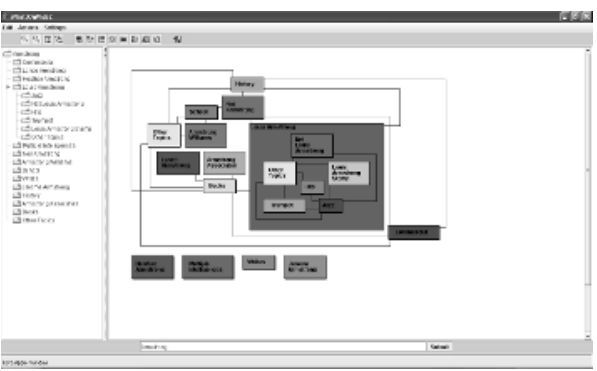

(b)

Fig. 5. (a) A map obtained from the one of Figure 4 by expanding the categories "Jazz", "School", and "Louis Armstrong Stamp"; this last category contains two URLs, labeled with their titles. (b) Another map where isolated categories of little relevance have been automatically hidden. 
not appear meaningful; this category is even completely unrelated with every other category.

- Automatic filtering. As the categories have a relevance score, also each relationship between two categories has an assigned weight that measures the strength of that relationship (see Section 2). The user can customize a visibility threshold both for the categories and for their relationships. The system automatically hides those edges whose weight is less than the given threshold, and it hides all categories that are isolated and whose score is less than the specified threshold. For example, in Figure 5(b) several isolated categories of the map in Figure 4 have been automatically hidden by the system, after that the user has increased the visibility threshold for the categories.

- Pruning of categories. The user can select a desired subset of categories in which she is no longer interested. After this selection, the system can be forced to only recompute the drawing, without changing the content of the remaining clusters, or it can be forced to also recompute the whole clusters hierarchy using the remaining documents. This pruning operation can be used to progressively reduce the amount of information that the user handles, and to refine the remaining information if necessary. For example, Figure 6 shows the result of a pruning of the categories "Neil Armstrong", "School", and "Armstrong Williams", starting from the map of Figure 5(b). After the pruning only the drawing is recomputed, in order to optimize the occupied area. In the map of Figure 6, three further categories are selected by the

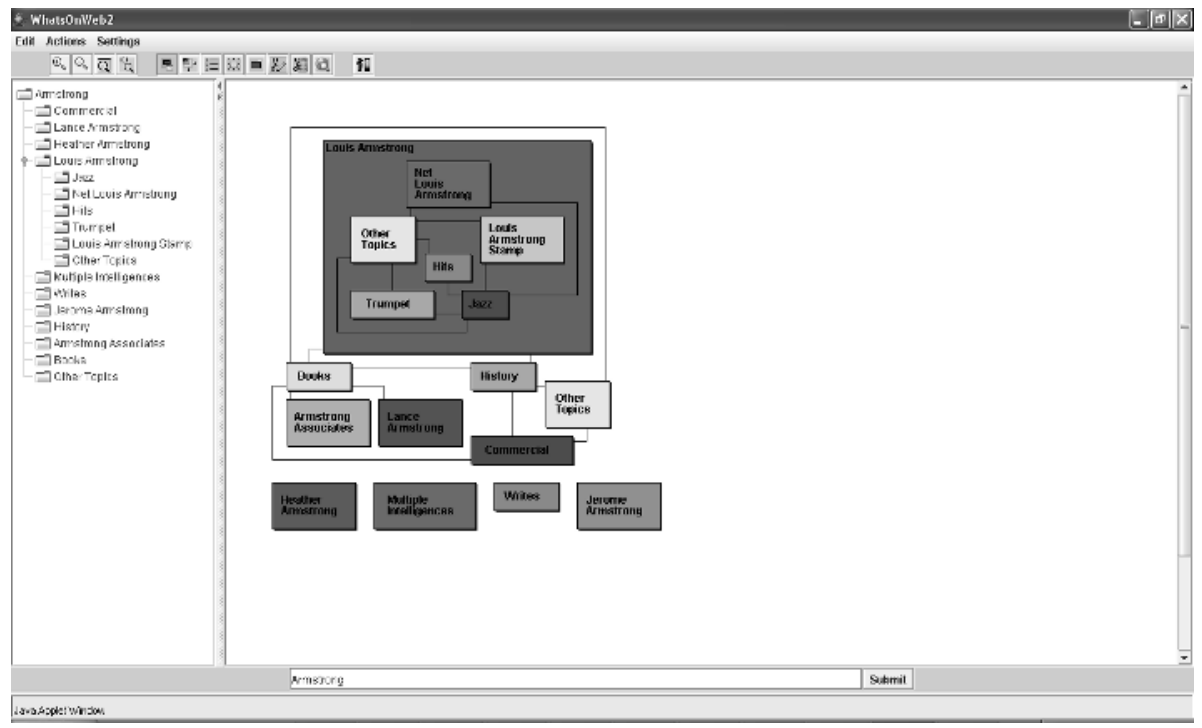

Fig. 6. The categories "Neil Armstrong", "School", and "Armstrong Williams" have been pruned from the map of Figure $5(\mathrm{~b})$, and only the drawing has been recomputed 
user to be pruned; in this case the user requires to completely recompute the clusters hierarchy after the pruning operation. The result is depicted in Figure 7, observe how the set of clusters is changed.

- Edge exploration. The user can explore the information associated with the edges of the map. If the mouse is positioned on an edge $(u, v)$, a tool-tip is displayed that shows both the weight and the complete list of strings that form the labels of the relationship between $u$ and $v$. Clicking on the edge,

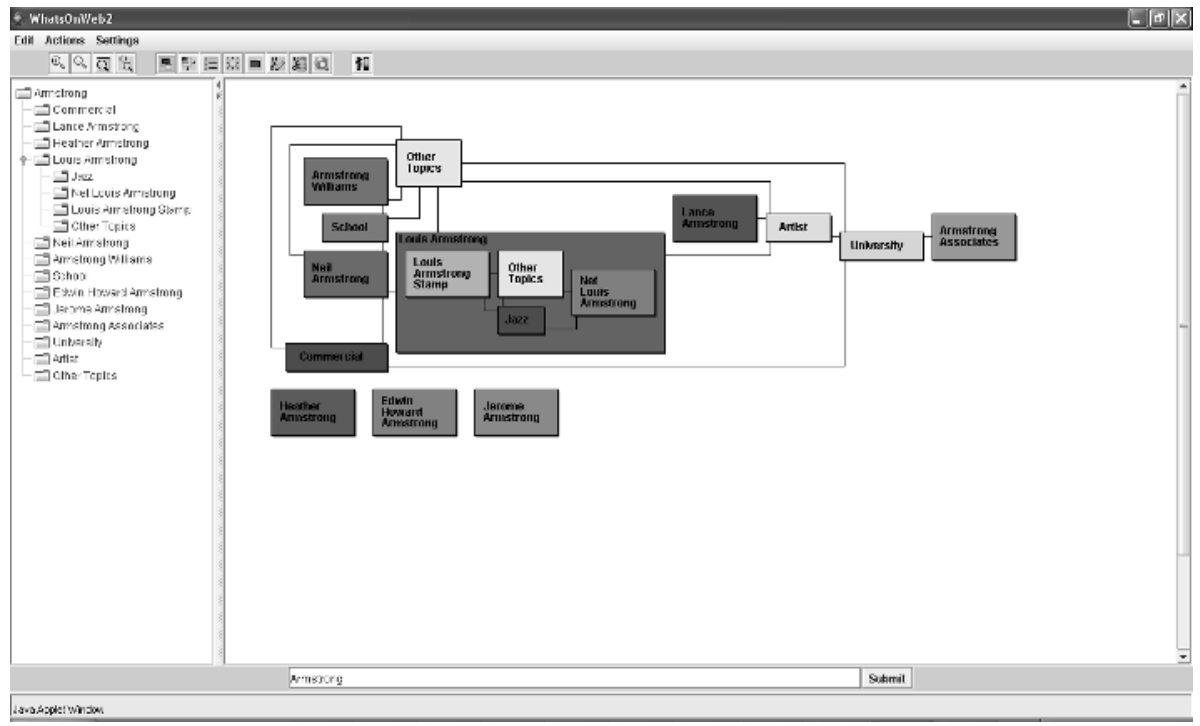

Fig. 7. The result of a further pruning of the categories "Hits", "Trumpet" and "Writes", where also the clustering is recomputed

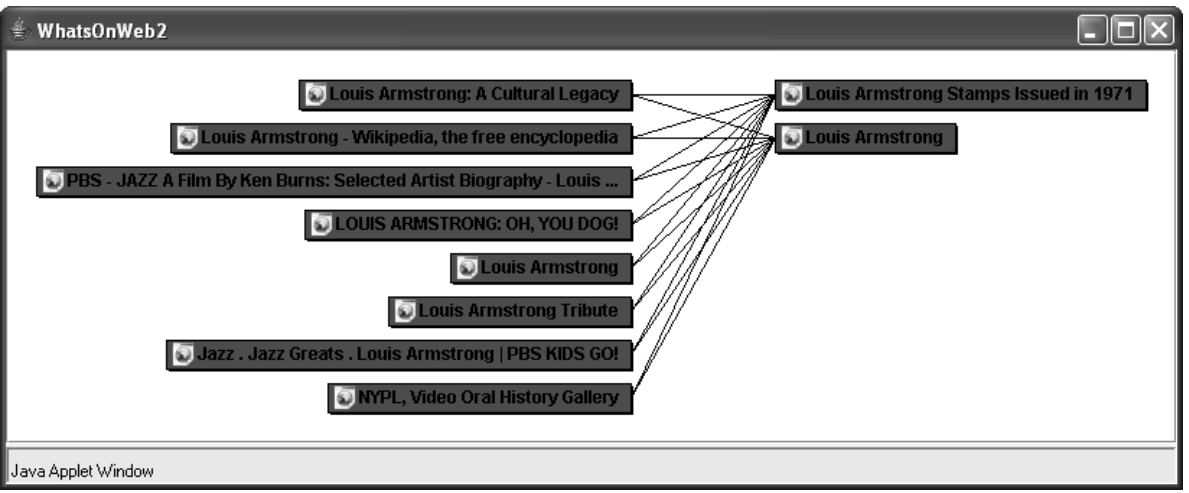

Fig. 8. A 2-layered drawing that shows the relationships between the URLs of "Louis Armstrong Stamp" and those of "Jazz" 
a 2-layered drawing is shown to the user, that displays the URLs in $u$, the URLs in $v$, and the relationships between these two sets of URLs, as they appear in the snippet graph (see Figure 8). This feature makes it possible to isolate all those documents that give rise to a specific relationship and can be also used as a tool to evaluate and tune the mechanism adopted by the system for creating cluster relationships.

- Query refinement. The user can select one or more categories and can ask the system to repeat the query by adding to the original query string the labels of the selected categories. In this way it is possible to obtain semantic categories that are more and more specific for the domain in which the user is interested.

\section{Implementation and Graph Drawing Engine}

The GUI environment and the client/server communication layer of WhatsOnWeb have been implemented using Java technology. From the client side we defined a Java Applet which interacts with the user and forwards the requests to the server. From the server side we defined several Java Servlets; one of these Servlets interacts with a graph drawing engine, which implements engineered versions of orthogonal graph drawing algorithms and which uses the facilities of the GDToolkit library 4. During the browsing of the user, the map displayed on the screen changes dynamically, depending on the expansion or contraction operations required by the user. Whats0nWeb has two main goals each time a new drawing must be computed: (i) The preservation of the user mental map, and (ii) the minimization of the area occupied by the drawing.

The first goal is achieved by computing an orthogonal shape for each subgraph of the super clustered graph $G^{\prime \prime}$ (see Subsection)and by always using the same shape each time a drawing of the subgraph must be displayed. To compute this shape the system first planarizes the subgraph and then uses the simple Kandisky model and the flow-based algorithm described in [1].

The second goal is achieved by determining the size of each super node in the map, and by applying the effective compaction algorithm for orthogonal drawings with vertices of prescribed size described in [3]. The compaction algorithm is recursively applied to all those orthogonal shapes (computed in the previous step) that must be visualized. More in detail, during the browsing of the user each cluster $\mu$ maintains a state that informs if it is expanded or contracted. If $\mu$ is contracted, it is represented as a "small" rectangle containing its label; all its subclusters are hidden. If $\mu$ is expanded, its super node $C_{\mu}$ is drawn as a rectangular region $r_{\mu}$ that contains the drawings of its subclusters and the label of $\mu$. The dimensions of $r_{\mu}$ depend on the state of the subclusters of $\mu$, which can be expanded or not. To determine the dimensions of $r_{\mu}$ we apply a procedure that recursively constructs a drawing $\Gamma$ of the subclusters of $\mu$ and then sets the height and the width of $r_{\mu}$ as the height and the width of the bounding box of $\Gamma$, plus a small area needed to place the label of $\mu$.

\footnotetext{
${ }^{4}$ http://www.dia.uniroma3.it/ $\sim$ gdt
} 
Finally, we implemented a standard Sugyiama algorithm (see, e.g., 4) in order to construct the 2-layered drawings that show the relationships between the URLs of two distinct clusters.

\section{References}

1. P. Bertolazzi, G. D. Battista, and W. Didimo. Computing orthogonal drawings with the minimum number of bends. IEEE Trans. on Comp., 49(8):826-840, 2000.

2. M. Brinkmeier. Communities in graphs. In Innovative Internet Community Systems (IICS'03), volume 2877 of LNCS, 2003.

3. G. Di Battista, W. Didimo, M. Patrignani, and M. Pizzonia. Orthogonal and quasi-upward drawings with vertices of prescribed sizes. In Graph Drawing (GD '99), volume 1731 of LNCS., pages 297-310, 1999.

4. G. Di Battista, P. Eades, R. Tamassia, and I. G. Tollis. Graph Drawing. Prentice Hall, Upper Saddle River, NJ, 1999.

5. E. Di Giacomo, W. Didimo, L. Grilli, and G. Liotta. A topology-driven approach to the design of web meta-search clustering engines. In SOFSEM '05, volume 3381 of LNCS., pages 106-116, 2005.

6. P. Ferragina and A. Gulli. The anatomy of a clustering engine for web-page snippet. In The Fourth IEEE International Conference on Data Mining (ICDM'04), 2004.

7. E. Hartuv and R. Shamir. A clustering algorithm based on graph connectivity. Information Processing Letters, 76:175-181, 2000.

8. M. E. J. Newman and M. Girvan. Finding and evaluating community structure in networks. Phys. Rev. E 69, 2004.

9. G. Salton. Automatic Text Processing. The Transformation, Analysis, and Retrieval of Information by Computer. Addison-Wesley, 1989.

10. O. Zamir and O. Etzioni. Web document clustering: A feasibility demonstration. In Research and Development in Information Retrieval, pages 46-54, 1998.

11. O. Zamir and O. Etzioni. Grouper: a dynamic clustering interface to web search results. Computer Networks, 31(11-16):1361-1374, 1999. 